\title{
A AVALIAÇÃO DA COMPOSIC̣ÃO CORPORAL - A MEDIÇÃO DE PREGAS ADIPOSAS COMO TÉCNICA PARA A AVALIAÇÃO DA COMPOSIC̣ÃO CORPORAL
}

AUTORES
Francisco Gonçalves ${ }^{1}$
Paulo Mourão ${ }^{2}$
${ }^{1}$ Licenciado e Doutorando em Educação Física
e Desporto pela Universidade de Trás-os-
-Montes e Alto Douro
${ }^{2}$ Licenciado em Educação Física pelo ISMAI
e Mestre em Ciências do Desporto pela
Universidade de Trás-os-Montes e Alto Douro

A AVALIAÇÃO DA COMPOSIC̣ÃO CORPORAL - A MEDIÇÃO DE PREGAS ADIPOSAS COMO TÉCNICA PARA A AVALIAC̄ÃO DA COMPOSIC̣ÃO CORPORAL 4(4): 13-21

\section{PALAVRAS-CHAVE}

composição corporal;

antropometria; pregas adiposas.

\section{KEYWORDS}

body composition; anthropomety; skinfold.

\section{RESUMO}

Com o nosso trabalho, pretendemos elaborar um documento de pesquisa e apoio, assim como de orientação para futuros "avaliadores" da Composição corporal, através da Antropometria e mais concretamente, através da medição de pregas adiposas.

Isto surge porque a análise detaIhada da composição corporal permite a quantificação de grande variedade de componentes corporais e torna-se de extrema importância pois permite determinar a quantidade total e regional de gordura corporal (Fragoso e Vieira, 2000).

Existem várias técnicas para a avaliação da mesma Composição Corporal, desde técnica directas, técnicas indirectas e técnicas duplamente indirectas. Logicamente, as técnicas directas são mais precisas, mas infelizmente são as mais dispendiosas e morosas, pelo que se tornam de difícil execução. Assim, torna-se bastante importante a existência das outras técnicas que são bem mais acessíveis, como é o caso da Antropometria e a medição de pregas adiposas. No entanto, mesmo nesta técnica existem cuidados e procedimentos, tais como o tipo de instrumento utilizado, os locais de medição, etc., que devem ser tidos em conta, de forma a minimizar erros de medida.

\section{ABSTRACT}

With our work, we intend to elaborate a document of research and support, as well as of orientation for future "appraisers" of the Body Composition, through the Anthropometry and more concretely, the measurement of Skinfolds.

This arise because the detailed analysis of the Body Composition allows the quantification of many body components and becomes of extreme importance therefore allows to determine the total and regional amount of body fat (Fragoso and Vieira, 2000).

Exists several techniques for the evaluation of the same Body Composition, since direct technique, indirect techniques and doubly indirect techniques. Logically, the direct techniques are more exactly, but unhappyly they are expensive and slow, for that, they become of difficult execution.

Thus, the existence of the other techniques becomes very important because are more accessible, as it is the case of the Anthropometry and the measurement of Skinfolds. However, even in this technique exists very cares and procedures, such as the type of used instrument, the places of measurement, etc., that must be had in account, to minimize measure errors. 


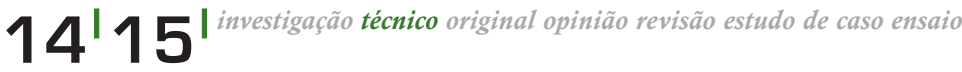

\section{INTRODUC̣ÃO}

A composição corporal é a proporção entre os diferentes componentes corporais e a massa corporal total, sendo normalmente expressa pelas percentagens de gordura e de massa magra. Pela avaliação da composição corporal, podemos, além de determinar os componentes do corpo humano de forma quantitativa, utilizar os dados obtidos para detectar o grau de desenvolvimento e crescimento de crianças e jovens e o estado dos componentes corporais de adultos e idosos (Heyward e Stolarczyk, 2000). A sua análise detalhada permite a quantificação de grande variedade de componentes corporais e torna-se de extrema importância porque permite determinar a quantidade total e regional de gordura corporal (Fragoso e Vieira, 2000).

É neste sentido, que a avaliação da Composição Corporal se revela como extraordinariamente importante. Existem várias formas de o fazer, no entanto, as mais precisas são bastante dispendiosas e morosas. Porém, existem formas indirectas de calcular a Composição Corporal, que são mais acessíveis a todos os níveis. Uma dessas formas é a Antropometria. No nosso trabalho, centramo-nos e aprofundamos uma técnica antropométrica para o cálculo da Composição Corporal, que é a Medição de Pregas Adiposas.

Assim, no nosso trabalho, começamos por descrever o que é a Composição Corporal, referindo a importância da avaliação da mesma. Seguidamente, iremos referir algumas formas de medição e avaliação da mesma, até chegarmos à Medição de Pregas Adiposas, onde iremos abordar entre outros, as fontes de erro de medida, a técnica de medição e os locais de medição padronizados.
Desta forma, esperamos que o nosso trabalho sirva de linha de orientação a futuros "avaliadores" da composição corporal, através da antropometria e mais concretamente, através da medição de pregas adiposas.

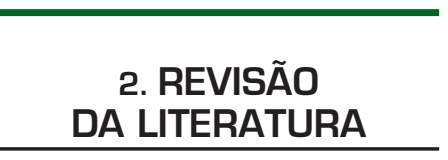

2.1. COMPOSIC̣ÃO CORPORAL

Segundo em Heyward e Stolarczyk (2000), a Antropometria tem sido utilizada para avaliar o tamanho e as proporções dos segmentos corporais, através da medição de circunferências e comprimento dos segmentos corporais. Por volta de 1915, a espessura do tecido adiposo subcutâneo foi medida utilizando-se o método das pregas adiposas. Nos anos 60 e 70, essas medidas foram utilizadas para desenvolver várias equações antropométricas para predizer a densidade corporal total e a gordura corporal.

Um outro método de avaliação da composição corporal aplicável em ambientes de campo e clínico é a análise da Bioimpedância. Esta técnica foi iniciou-se no início dos anos 60, e é um método que avalia essencialmente a quantidade de água total no organismo, através da aplicação de uma corrente eléctrica, baseando-se no princípio de que só as substâncias ionizadas têm, de conduzir corrente eléctrica. Caracteriza-se por ser um método rápido e não invasivo, porém é mais caro que o método das pregas adiposas e antropométrico. De acordo com Fragoso e Vieira (2000) existe ainda a densitometria radiológica de dupla energia, sendo um método não invasivo que se utiliza para medir o conteúdo mineral ósseo, a quantidade de gordura corporal e a quantidade de massa isenta de gordura feita através da quantificação da quantidade de feixe de raio-x que é retardado ao atravessar aquelas superfícies.

Actualmente, e segundo Heyward e Stolarczyk (2000), a composição corporal é a proporção entre os diferentes componentes corporais e a massa corporal total, sendo normalmente expressa pelas percentagens de gordura e de massa magra. Pela avaliação da composição corporal, podemos, além de determinar os componentes do corpo humano de forma quantitativa, utilizar os dados obtidos para detectar o grau de desenvolvimento e crescimento de crianças e jovens e o estado dos componentes corporais de adultos e idosos.

Segundo Fragoso e Vieira (2000), quando falamos em composição corporal referimo-nos ao estudo de diferentes componentes químicos do corpo humano. A sua análise detalhada permite a quantificação de grande variedade de componentes corporais, tais como a água, as proteínas, a gordura, os hidratos de carbono, os minerais, etc., apesar das proporções corporais relativas destes componentes serem idênticas em todos os indivíduos, sendo o maior constituinte corporal a água, seguindo-se as proteínas e as gorduras, os hidratos de carbono, os minerais e os outros componentes, a quantidade de cada constituinte corporal varia de indivíduo para indivíduo.

\subsubsection{IMPORTÂNCIA DA MEDIC̣ÃO DA COMPOSIĈ̃̃ CORPORAL}

Em termos de condição física, torna-se primordial a medição da composição corporal, porque esta 
avalia a quantidade total e regional de gordura corporal. Segundo Heyward e Stolarczyk (2000), podemos utilizar a composição corporal para:

- Identificar riscos de saúde associados a níveis excessivamente altos ou baixos de gordura corporal total;

- Identificar riscos de saúde associados ao acumulo excessivo de gordura intra-abdominal;

- Proporcionar a percepção sobre os riscos de saúde associados à falta ou ao excesso de gordura corporal;

- Monitorizar mudanças na composição corporal associadas a certas doenças;

- Avaliar a eficiência das intervenções nutricionais e de exercícios físicos na alteração da composição corporal;

- Estimar o peso corporal ideal de atletas e não atletas;

- Formular recomendações dietéticas e prescrições de exercícios físicos;

- Monitorizar mudanças na composição corporal associadas ao crescimento, desenvolvimento, maturação e idade.

Katch e McArdle (1983), demonstraram que a percentagem de gordura corporal (avaliada através das pregas adiposas] de rapazes e raparigas, em idades compreendidas entre 5 a 18 anos, estava correlacionada com factores de risco de doença coronária (pressão sanguínea, colesterol total e proporção das lipoprotaínas) em crianças e adolescentes. Este, pode ser um dos factores que levam a que a avaliação da composição corporal, deva ser incluída no currículo de Educação Física (Heyward e Stolarczyk, 2000].

\subsubsection{MÉTODOS DE AVALIAC̣ÃO DA COMPOSIC̣ÃO CORPORAL}

Existem várias técnicas para a determinação da composição corporal, chamando-se a estas métodos. Estes procedimentos podem-se classificar em (Pontes, 2003):

\subsubsection{MÉTODO DIRECTO}

É aquele em que há separação e pesagem de cada um dos componentes corporais isoladamente.

De acordo com Costa (2001), a dissecação de cadáveres é a única metodologia considerada directa. Neste método procede-se à separação dos diversos componentes estruturais do corpo humano, com o intuito de verificar a sua massa isoladamente e estabelecer relações entre os diversos componentes e a massa corporal total. Desta forma, podemos perceber a razão pela qual há tão poucos estudos tendo como base este método, pois é uma metodologia de difícil consecução.

\subsubsection{MÉTODOS INDIRECTOS}

São aqueles em que não há manipulação dos componentes separados, mas utilizam-se princípios químicos e físicos que visam a extrapolação das quantidades de gordura e de massa magra;

Estes métodos são aqueles em que não há manipulação dos componentes separados, pois realizam as estimativas a partir de princípios químicos e físicos com base na extrapolação das quantidades de gordura e de massa magra.

Entre os métodos indirectos, a Pesagem Hidrostática tem sido considerada como referência para a validação de métodos duplamente indirectos. Esta baseia-se no Princípio de Arquimedes segundo o qual "todo o corpo mergulhado num fluido [líquido ou gás) sofre, por parte do fluido, uma força vertical para cima, cuja intensidade é igual ao peso do fluido deslocado pelo corpo". A Pesagem Hidrostática define o volume corporal pelo cálculo da diferença entre a massa corporal aferida normalmente e a medição do corpo submerso em água. Na Pesagem Hidrostática, primeiro verifica-se a massa do indivíduo fora de água, e seguidamente dentro de água. Na pesagem dentro de água, o indivíduo deve realizar uma expiração máxima, visando eliminar a maior quantidade de ar possivel dos pulmões. Como este procedimento envolve adaptação ao meio líquido, são realizadas de 8 a 12 pesagens submersas, apresentando, na fórmula a média das três maiores medidas. Outro dos métodos indirectos que tem sido utilizado para validação dos métodos duplamente indirectos é a Densitometria Radiológica de Dupla Energia. Este método é considerado como uma técnica avançada para avaliar a composição corporal [Costa, 2001).

Segundo Fragoso e Vieira (2000) este método é um método não invasivo que se utiliza para medir o conteúdo mineral ósseo, a quantidade de gordura corporal e a quantidade de massa livre de gordura.

0 princípio básico em que se fundamenta é o de que, o osso e os tecidos moles do corpo podem ser atravessados, até uma profundidade de $30 \mathrm{~cm}$, por feixes de raio-x com energias diferentes, emitidos alternadamente, sendo a predição do conteúdo mineral ósseo, da massa gorda e da massa livre de gordura feita através da quantificação da quantidade de feixe de raio-x que é retardado ao atravessar aquelas superfícies. 


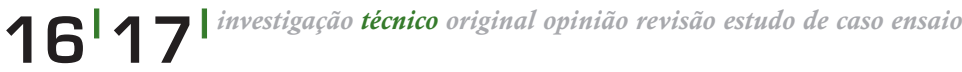

\subsubsection{MÉTODOS \\ DUPLAMENTE INDIRECTOS}

São aqueles que surgem a partir dos métodos indirectos e que se encontram devidamente validados.

Os procedimentos duplamente indirectos podem ser validados por um método indirecto, mais frequentemente a Pesagem Hidrostática e a Densitometria Radiológica de Dupla Energia, sendo que os mais utilizados em estudos de campo são, nos dias de hoje, a Bioimpedância e a Antropometria.

A análise de impedância bioeléctrica (Bioimpedância) é um método rápido, não-invasivo e relativamente barato para avaliar a composição corporal em situações de campo e clínicas. Uma corrente eléctrica de baixo nível é passada através do corpo do indivíduo e a impedância, ou oposição ao fluxo da corrente, é medida com um analisador de Bioimpedância. A resistência ao fluxo da corrente será maior em indivíduos com grande quantidade de gordura corporal, dado que o tecido adiposo é mau condutor de corrente eléctrica pela sua relativa baixa quantidade de água. Existe uma forte relação entre as medidas de impedância total do corpo e água corporal total, por este motivo sugere-se que o método de Bioimpedância seja uma ferramenta valiosa para a análise da composição corporal e avaliação da água corporal total no ambiente clínico (Heyward e Stolarczyk, 2000).

No entanto, para a avaliação clínica ou para o estudo de grandes grupos populacionais surgem diversas dificuldades com a utilização de técnicas indirectas. Como tal, surgem alternativas para minimizar essas mesmas dificuldades. Uma das alternativas mais comuns é o uso de algumas técnicas baseadas na utilização de medidas antropométricas. Estas técnicas incluem proporções massa - estatura, perímetros corporais e medidas de pregas adiposas.

\subsection{ANTROPOMETRIA}

De acordo com Costa (2001), devido ao baixo custo operacional e à relativa simplicidade de utilização, os métodos antropométricos são aplicáveis a grandes amostras e podem proporcionar estimativas nacionais e dados para análise de mudanças.

A predição da composição corporal através da Antropometria utiliza medidas relativamente simples como massa, estatura, perímetros, diâmetros ósseos e espessura das pregas adiposas. Quando o objectivo é estimar somente a percentagem de gordura corporal, as medidas mais utilizadas são as pregas adiposas. A Antropometria pode ser usada para identificar indivíduos em risco de doença, sendo indicada para pesquisas epidemiológicas de larga escala e propósitos clínicos (Nobre, 1995). As medidas de perímetros e diâmetros ósseos são indicadores de massa corporal magra, enquanto que, alguns perímetros são altamente associados à componente de gordura. Isso confirma que as medidas de circunferência reflectem tanto a gordura, quanto a massa livre de gordura da composição corporal.

Com base em Heyward e Stolarczyk (2000), a exactidão e a fidelidade das medidas antropométricas podem ser afectadas por:

- Equipamento;

- Habilidade do Avaliador;

- Factores Individuais;

- Equação de predição utilizada.

Um outro método de avaliação da composição corporal é o Índice de
Massa Corporal. Este método é definido pela proporção do peso do corpo para altura ao quadrado (Heyward e Stolarczyk, 2000). 0 Índice de Massa Corporal é um método de grande importância prática e mostra uma boa correlação com a mortalidade e morbilidade gerais e com a mortalidade e morbilidade relacionadas com diversas patologias.

Uma outra medida antropométrica com potencial para prognosticar a distribuição de gordura e risco de doença. 0 Índice de Conicidade é baseado na ideia de que o corpo humano muda de formato de um cilindro para o de um cone duplo, com o acumulo de gordura ao redor da cintura.

\subsubsection{PREGAS ADIPOSAS}

A medição das pregas adiposas, também denominadas pregas de gordura subcutânea ou skinfold, constitui um dos métodos de avaliação da gordura corporal mais utilizados, pela facilidade de utilização, baixo custo e pela sua grande correlação com a gordura corporal total [Fragoso e Vieira, 1994). Esta metodologia baseia-se no facto de cerca de $50 \%$ da gordura corporal total estar localizada subcutaneamente, constituindo aquilo que se designa por massa adiposa subcutânea ou panículo adiposo. Em virtude da espessura da pele representar apenas cerca de 1,8 mm, a maioria da espessura da prega é representativa de gordura subcutânea (Moreira, 1995).

Pesquisas demonstram que a gordura subcutânea, avaliada pelo método das pregas adiposas em doze locais, é similar ao valor obtido nas imagens de ressonância magnética (Heyward Stolarczyk, 2000). 
A medição das pregas adiposas possui uma elevada correlação com os outros meios mais sofisticados de avaliação da composição corporal. Para além disto, constitui uma alternativa simples, menos dispendiosa e precisa (Moreira, 1995).

Para Ferreira (1998), a espessura das pregas subcutâneas quer no braço (tricipital) quer no tronco [subescapular], permitem uma avaliação mais específica para a obesidade ou sobrecarga ponderal, do que a utilização isolada do Índice de Massa Corporal.

\subsubsection{FONTES DE ERRO DE MEDIDA}

Para Heyward e Stolarczyk (2000), a exactidão teórica das equações de pregas adiposas para predizer a Densidade Corporal é 0,0075 $\mathrm{g} / \mathrm{cm}^{3}$ ou 3,3 Gordura Corporal devido à variabilidade biológica em estimar a gordura subcutânea através da espessura das pregas adiposas e diferenças inter-individuais na relação entre a gordura subcutânea e a gordura corporal total. Portanto, erros de predição $\leq 3,5 \%$ Gordura Corporal ou $\leq 0,0080 \mathrm{~g} / \mathrm{cm}^{3}$ para equações de pregas adiposas são aceitáveis, porque uma parte desse erro é atribuída ao método de referência. Na estimativa da Gordura Corporal, a validade e fiabilidade das medidas e do método das pregas adiposas são afectadas pelos seguintes factores: (Heyward Stolarczyk, 2000):

- Habilidade do avaliador;

- Aproximadamente 3 a 9\% da variabilidade em medidas de pregas adiposas podem ser atribuída a erro de medida devido a diferenças entre avaliadores. Para que a diferença seja a menor possivel, é necessário que haja uma padronização dos procedimentos do teste (marcação do local da prega e bom conhecimento das localizações anatómicas].
- Tipo do adipómetro:

- As pregas de adiposidade subcutânea são medidas através de um instrumento designado de adipómetro, cujas normas de construção estão padronizadas. De entre os mais utilizados, destacam-se os adipómetros de Lange e de Harpenden. Contudo, face ao seu custo elevado custo, têm aparecido no mercado adipómetros fabricados em material plástico, mais baratos, mais leves e cuja a precisão de medida tem merecido uma atenção crescente por parte dos seus fabricantes à semelhança do que acon-tece com o adipómetro de Slimguide. Seja qual for o tipo de adipómetro utilizado, é importante ter em atenção que os mesmos são susceptíveis de conduzirem a diferentes resultados, contudo, todos eles devem exercer um a pressão constante de $10 \mathrm{~g} / \mathrm{mm}^{2}$ sobre a pele e permitirem leituras até às décimas de milímetro. De salientar que o adipómetro utilizado ao longo do estudo deve ser sempre o mesmo. (Heyward e Stolarczyk, 2000; Claessens, Beunen e Malina, s/d).

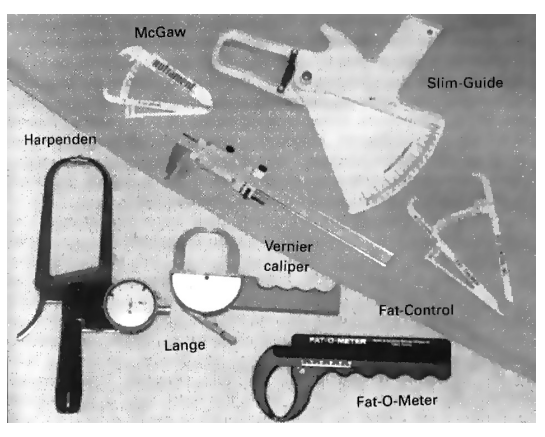

FIGURA 1

Adipómetros de alta precisão e de plástico (Heyward e Stolarczyk, 2000).
- Factores individuais:

- A variabilidade em medidas das pregas adiposas entre indivíduos pode ser atribuída não apenas à diferença na quantidade de gordura subcutânea no local, mas à diferença na espessura da pele, compressibilidade do tecido adiposo, manuseio e nível de hidratação. Para tal é necessário ter alguns cuidados. Assim, as pregas adiposas não devem ser medidas:

- Imediatamente após o exercício, devido à mudança dos fluidos corporais para a pele tender a aumentar o tamanho prega;

- Nas mulheres, durante período menstrual, devido à retenção de água que aumenta a espessura da prega;

- Dos dois lados (direito/esquerdo). Ainda não existe um padrão sobre qual o lado do corpo, em que as medidas antropométricas devem ser medidas. Segundo Harrisson et al. (1988), estas devem ser medidas do lado direito do corpo.

- Equação de predição utilizada:

- As equações de predição devem ser seleccionadas baseadas na idade, sexo, etnia e nível de actividade física.

\subsubsection{TÉCNICA DE MEDIC̣ÃO}

Para se desenvolver a habilidade como avaliador de pregas adiposas, é necessário muito tempo e prática. Seguir procedimentos padronizados aumenta a exactidão e fidelidade das medidas (Harrisson et al., 1988; Heyward e Stolarczyk, 2000; Claessens, Beunen e Malina, $s / d)$. 


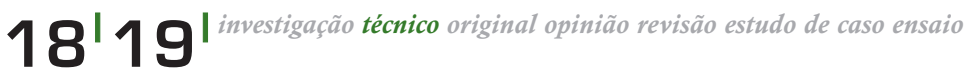

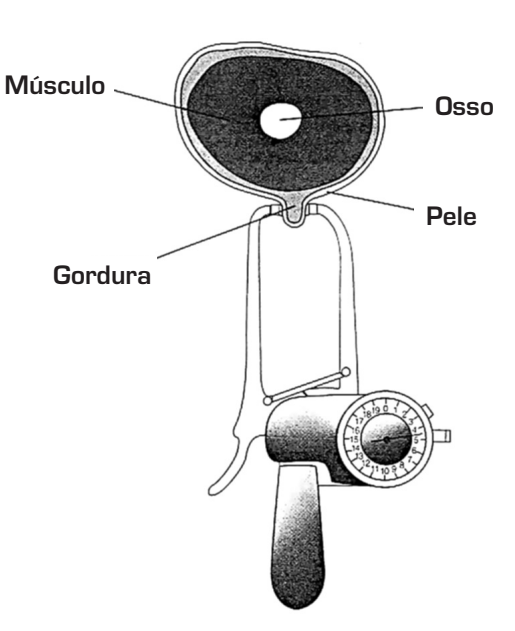

FIGURA2

Anatomia de uma prega adiposa (adaptado de Heyward e Stolarczyk, 1996).

Segundo Moreira (1995), a técnica de medição deve respeitar os seguintes procedimentos:

- Efectuar todas as medidas das pregas adiposas do lado direito do corpo;

- A temperatura ambiente deve situar-se entre os 18 e os $22{ }^{\circ} \mathrm{C}$ e a humidade deve ser inferior a 60\%;

- Cuidadosamente identificar, medir e marcar o local das pregas adiposas, especialmente tratando-se de um avaliador inexperiente;

- Para a medição, é preciso definir-se o eixo maior da prega e esta deve ser segura, firmemente, entre o polegar e o indicador da mão esquerda. A prega é destacada $1 \mathrm{~cm}$ acima do local a ser medido;

- Destacar a prega, colocando o polegar e o indicador a uma distância de $8 \mathrm{~cm}$, numa linha perpendicular ao eixo longo da prega. É nas extremidades destes $8 \mathrm{~cm}$ [4+4] que a elevação da prega vai ser realizada. 0 eixo longo é paralelo em relação às linhas naturais da pele. Entretanto, para indivíduos com pregas adiposas extremamente grandes, o polegar e o indicador necessitam de separar mais de $8 \mathrm{~cm}$ para que se consiga destacá-la;

- Manter a prega pressionada enquanto a medida é realizada;

- Colocar as hastes do adipómetro perpendiculares à prega, aproximadamente $1 \mathrm{~cm}$ abaixo do polegar e do indicador, e soltar a pressão das hastes lentamente;

- Efectuar as medições das pregas adiposas 4 segundos após a pressão ter sido aplicada para haver estabilização do ponteiro do adipómetro;

- Afastar as hastes do adipómetro para removê-lo do local. Fechar as hastes lentamente para prevenir danos ou perda da calibragem.

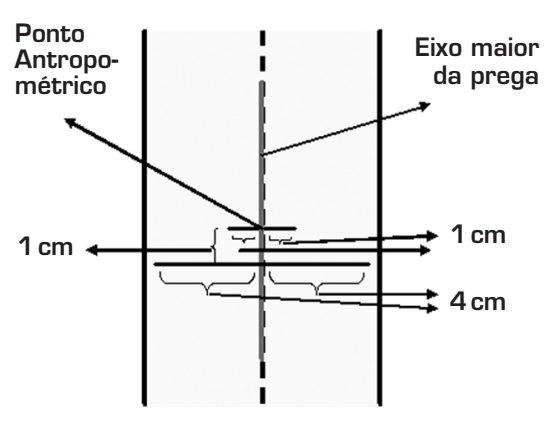

\section{FIGURA3}

Representação esquemática da marcação do local de medição.

Para aumentar a habilidade do avaliador de pregas adiposas há aspectos que devem ser levados em conta, como por exemplo:

- Ser meticuloso ao localizar os pontos anatómicos usados para identificar o local da prega, ao medir a distância e no momento de marcar o local com uma caneta;

- Ler o mostrador do adipómetro ao 0,1 mm mais próximo (Harpenden ou Holtain), 0,5 mm (Lange) ou $1 \mathrm{~mm}$ (adipómetros plásticos);

- Efectuar no mínimo de duas medidas para cada local. Se os valores diferem em mais de +/$10 \%$, efectuar medidas adicionais;

- Efectuar medidas de pregas adiposas numa ordem rotativa, em vez de leituras consecutivas em cada local;

- Efectuar medidas das pregas adiposas, quando a pele do indivíduo estiver seca e sem cremes;

- Praticar as medidas de pregas adiposas em 50 a 100 indivíduos;

- Evitar usar adipómetros plásticos no caso de se tratar de um avaliador inexperiente;

- Treinar com avaliadores experientes e comparar seus resultados;

- Usar um vídeo sobre medidas de pregas adiposas que demonstre a técnica correcta;

- Procurar treino adicional.

Após a marcação dos pontos de referência, Bubb (1986), refere que o procedimento mais adequado é realizar sucessivamente a medição dos vários skinfolds (pregas adiposas) por 2 ou 3 vezes, confrontando-se então os resultados obtidos. Para Jackson (1984), devem ser efectuadas no mínimo 2 medições em cada local e, no caso destas variarem em mais de $1 \mathrm{~mm}$, efectuar então uma terceira medição. Se os valores obtidos se forem tornando mais pequenos nas sucessivas medições, então isso é indicativo de que a gordura já está a ser comprimida, o que aliás é muito frequente em pessoas com muito tecido muscular. 
0 indivíduo que é alvo da medição deve estar em posição antropométrica, caracterizada do seguinte modo (Moreira, 1995):

- Posição vertical com braços pendentes ao lado do tronco e palmas das mãos em contacto com a face lateral das coxas (Fragoso e Vieira, 2000), as palmas das mãos do indivíduo devem estar voltadas para a frente, com os polegares voltados para fora e os restantes dedos para baixo;

- A cabeça deve estar no plano de Frankfurt, que pode ser determinado adoptando os seguintes procedimentos:

- Localizar o ponto Orbitale, localizado na margem inferior da cavidade orbitaria;

- Localizar o ponto Tragion, situado ao nível do pavilhão auricular, por cima do osso;

- Considerar uma linha imaginária unindo os dois pontos definidos anteriormente. Essa linha imaginária corresponde, de forma quase exacta, ao eixo do olhar quando o indivíduo tem os seus olhos dirigidos para a frente.

- 0 indivíduo deve estar descalço, com os calcanhares unidos, formando um ângulo de $60^{\circ}$ entre si e os dedos dos pés devem apontar para a frente.

\subsubsection{LOCAIS DE MEDIC̣ÃO DAS PREGAS ADIPOSÁS}

Os locais de medição estão devidamente normalizados e mesmo pequenas modificações na sua determinação são susceptíveis de alterar os resultados obtidos de uma forma significativa (Harrisson et al. 1988).

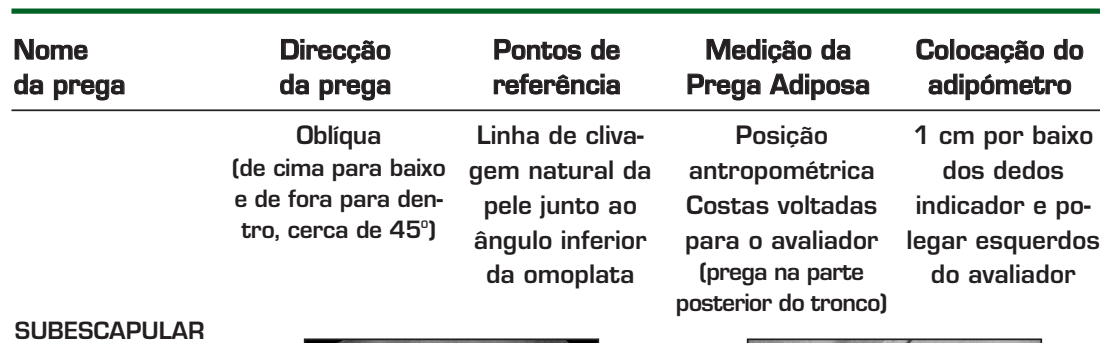

SUBESCAPULAR
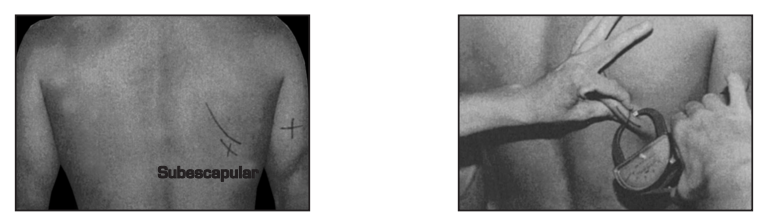

\begin{tabular}{|c|c|c|c|}
\hline $\begin{array}{c}\text { Oblíqua } \\
\text { [de cima para } \\
\text { baixo e de fora } \\
\text { para dentro] }\end{array}$ & $\begin{array}{l}\text { Prega axilar } \\
\text { anterior } \\
\text { mamilo }\end{array}$ & $\begin{array}{l}\text { Masculino - meia } \\
\text { distância entre a } \\
\text { prega axilar ante- } \\
\text { rior e o mamilo; } \\
\text { Feminino - 2/3 }\end{array}$ & $\begin{array}{l}1 \mathrm{~cm} \text { por baixo } \\
\text { dos dedos } \\
\text { indicador e po- } \\
\text { legar esquerdos } \\
\text { do avaliador }\end{array}$ \\
\hline
\end{tabular}

PEITORAL

da distância

do mamilo
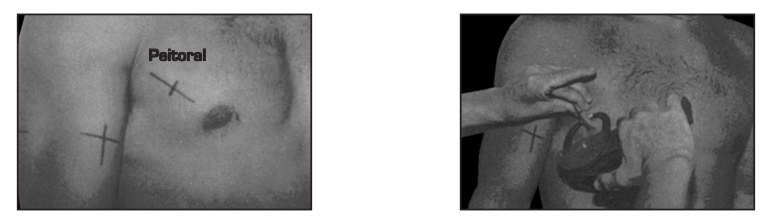

Horizontal

Linha midaxilar
Apófise xifoide
do externo

Sobre a linha

midaxilar na

$1 \mathrm{~cm}$ à frente 1 dos dedos legar esquerdo

MIDAXILAR

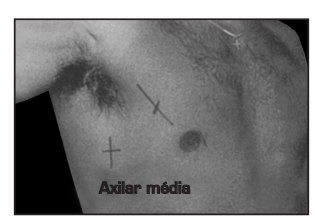
do avaliador xifoide do externo

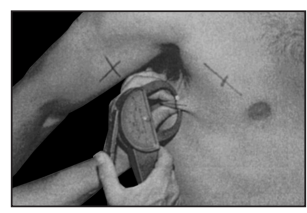

Oblíqua (de cima para baixo e de fora para dentro]

Lidaxila da crista iliaca

SUPRAILIACA

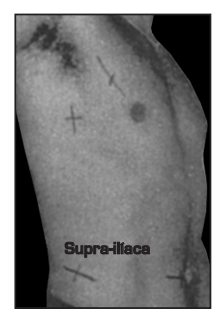

$2 \mathrm{~cm}$ de distância $1 \mathrm{~cm}$ à frente da linha midaxilar dos dedos sobre a crista ilía- indicador e poca, seguindo a cli- legar esquerdos vagem natural da do avaliador pele e formando um ângulo de $45^{\circ}$ com a horizontal

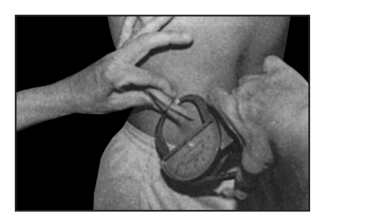

QUADRO1 (CONT.) Medição das várias Pregas Adiposas (Adaptado de Moreira, 1995). 


\begin{tabular}{lcccc}
\hline $\begin{array}{l}\text { Nome } \\
\text { da prega }\end{array}$ & $\begin{array}{l}\text { Direcção } \\
\text { da prega }\end{array}$ & $\begin{array}{c}\text { Pontos de } \\
\text { referência }\end{array}$ & $\begin{array}{c}\text { Medição da } \\
\text { Prega Adiposa }\end{array}$ & $\begin{array}{c}\text { Colocação do } \\
\text { adipómetro }\end{array}$ \\
\hline Vertical ou & Umbigo & $\begin{array}{c}5 \mathrm{~cm} \text { de distância } \\
\text { do centro }\end{array}$ & $\begin{array}{c}1 \mathrm{~cm} \text { distal dos } \\
\text { dedos polegar e }\end{array}$ \\
horizontal & & & $\begin{array}{c}\text { do umbigo e do do } \\
\text { lado do mesmo }\end{array}$ & $\begin{array}{c}\text { indor esquer- } \\
\text { dos do avaliador }\end{array}$
\end{tabular}

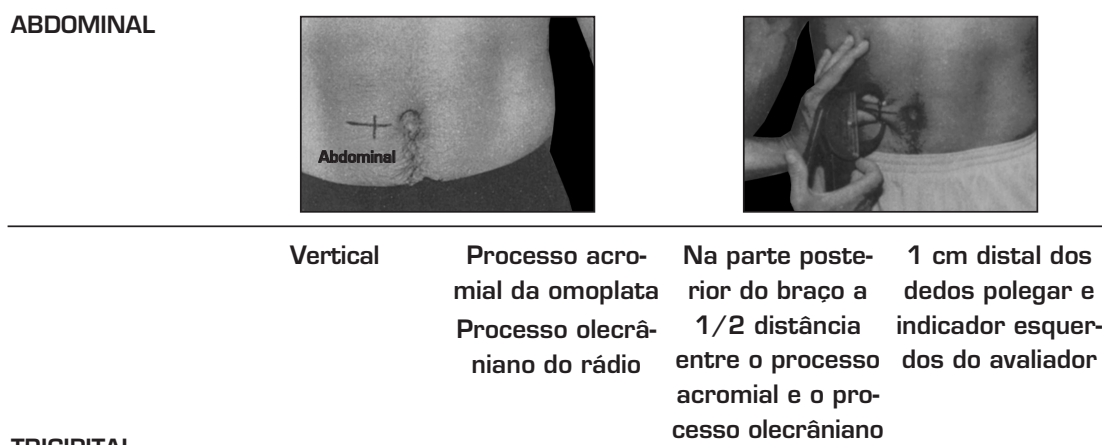

TRICIPITAL

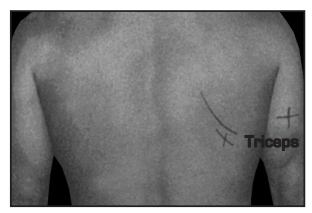

Vertical

Músculo bicípide
Zona de maior vo- $1 \mathrm{~cm}$ distal dos lume do músculo dedos polegar e bicipede a meia indicador esquer- distância entre dos do avaliador processo acro-
mial e o processo
olecrâniano

BICIPITAL

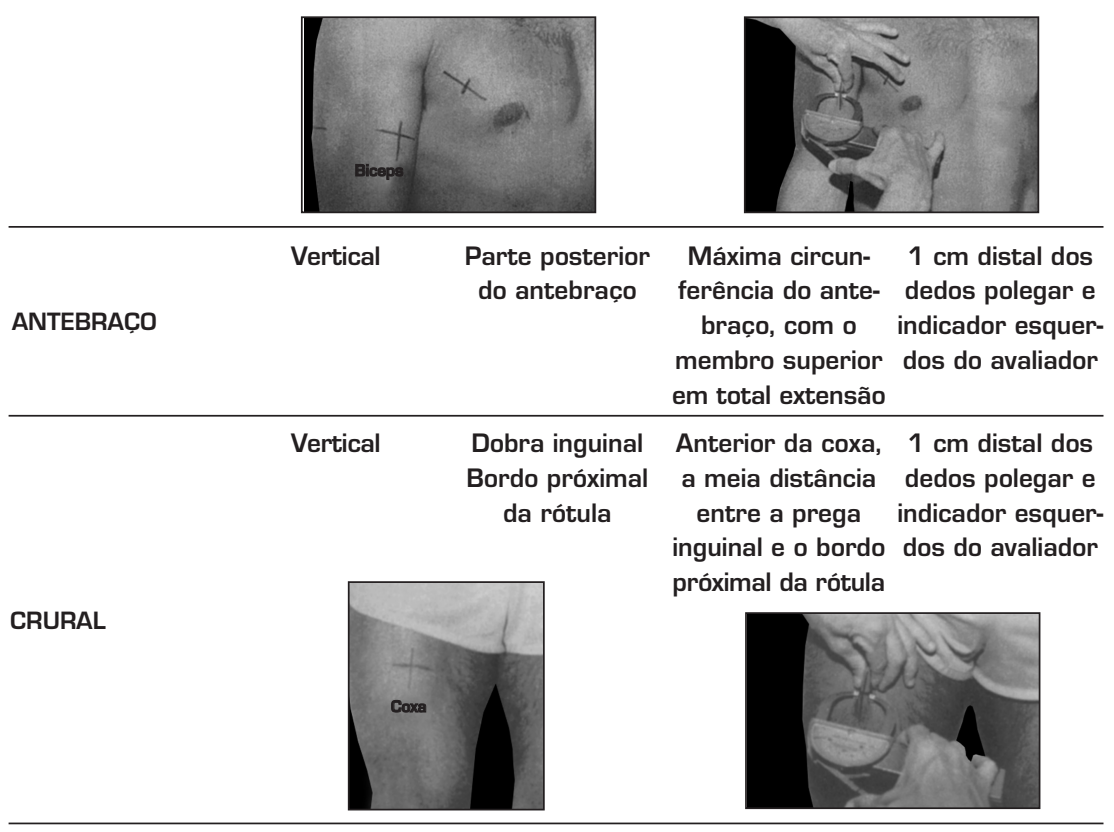

Fragoso e Vieira (2000), defendem que a prega tricipital é a mais útil para avaliar a percentagem global de gordura corporal e obesidade periférica, enquanto a prega subescapular avalia perfeitamente a gordura no tronco. Neste contexto o quociente entre ambas é um bom indicador do predomínio da obesidade em uma ou outra localização. Um quociente elevado indica a existência de uma obesidade central, que se correlaciona mais estreitamente com as alterações dos lípidos plasmáticos, arteriosclerose, patologias cardiovasculares e hipertensão.

\section{CONCLUSÕES}

Ao longo deste trabalho, procuramos focar a importância da avaliação morfológica bem como as diversas formas de o fazer. Centramos o nossa investigação numa das diversas formas de medir e avaliar a Composição corporal, que foi a avaliação antropométrica, mais concretamente através da medição de pregas adiposas.

Focamo-nos nesta área específica pela extrema facilidade e utilidade, relativamente às outras técnicas. Esta forma de medição, sendo mais acessivel e de mais fácil aplicação, torna-se num instrumento muito utilizado.

Desta forma, esperamos que o nosso trabalho sirva de linha de orientação a futuros "avaliadores" da composição corporal, através da antropometria e mais concretamente, através da medição de pregas adiposas. 


\section{CORRESPONDÊNCIA}

Francisco Gonçalves

Travessa Comendador Seabra da

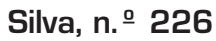

3720-297 Oliveira de Azeméis

E-mail: franciscojmg@gmail.com xicoze7@hotmail.com

TIms: 917668858 966833562

TIf: 256285335

\section{REFERÊNCIAS}

1. Bubb WJ [1986]. Relative Leanness. In Howley ET, Franks BD [Eds]. Health/Fitness Instructor's Handbook. (pp. 51-79). Champaign, Illinois: Human Kinetics Publishers.

2. Claessens $A L$, Beunen $G \&$ Malina RM [s/d). Anthropometry, Physique, Body Composition and Maturity. Paediatric Exercise Science and Medicine, 11-22.

3. Costa R [2001). Composição corporal - Teoria e Prática da Avaliação. Editora Manole.

4. Ferreira I (1998). Sensibilidade e Especificidade de Variáveis de Aptidão Física e Composição Corporal Como indicadores de Saúde em Jovens dos 10 aos 15 Anos de Idade. Tese não publicada, Universidade Técnica de Lisboa - Faculdade de Motricidade Humana, Lisboa, Portugal.

\begin{tabular}{|c|c|c|c|c|}
\hline $\begin{array}{l}\text { Nome } \\
\text { da prega }\end{array}$ & $\begin{array}{l}\text { Direcção } \\
\text { da prega }\end{array}$ & $\begin{array}{l}\text { Pontos de } \\
\text { referência }\end{array}$ & $\begin{array}{c}\text { Medição da } \\
\text { Prega Adiposa }\end{array}$ & $\begin{array}{c}\text { Colocação do } \\
\text { adipómetro }\end{array}$ \\
\hline \multirow{4}{*}{ GEMINAL } & Vertical & $\begin{array}{l}\text { Face interna } \\
\text { da perna }\end{array}$ & $\begin{array}{l}\text { Máximo volume } \\
\text { geminal da face } \\
\text { interna da perna }\end{array}$ & $\begin{array}{l}1 \mathrm{~cm} \text { distal dos } \\
\text { dedos polegar e } \\
\text { indicador esquer- } \\
\text { dos do avaliador }\end{array}$ \\
\hline & & & & \\
\hline & \multicolumn{4}{|c|}{ QUADR01 (CONCLUSÃo) } \\
\hline & \multicolumn{4}{|c|}{ Medição das várias Pregas Adiposas (Adaptado de Moreira, 1995). } \\
\hline
\end{tabular}

5. Fragoso I \& Vieira F (2000). Morfologia e Crescimento - Curso Prático. Edições FMH.

6. Harrisson GG, Buskirk ER, Carter JL, Johnston FE, Pollock ML, Roche AF \& Wilmore J (1988). Skinfold thicknesses and measurement technique. In Lohman T, Roche AF \& Martorell R (Eds). Anthropometric Standardization Reference Manual, (pp. 55-70). Champaign, Illinois: Human Kinetics Books.

7. Heyward V \& Stolarczyk L (1996) Applied Body Composition Assessment. Champaign, Illinois: Human Kinetics Books.

8. Heyward V \& Stolarczyk L (2000) Avaliação da Composição Corporal Aplicada. Editora Manole.

9. Jackson AS (1984). Pratical Methods of Measuring Body Composition. In Storlie J, Jordan HA (Eds). Evaluation and Treatment of Obesity. (pp. 93-111). Champaign.
Illinois: Life and Enhancement Publications.

10. Katch F \& McArdle W (1983). Nutrição, Controlo de Peso e Exercício. Editora Medsi.

11. Moreira MH (1995). Avaliação das Pregas Adiposas. Prova de Aptidão. Universidade de Trás-os-Montes e Alto Douro: Vila Real.

12. Nobre V (1995). Instrumentos, Técnicas de Promoção, Avaliação e Prescrição de Actividade Física de Jovens e Adultos Aparentemente Saudáveis. Tese não publicada, Universidade Técnica de Lisboa Faculdade de Motricidade Humana, Lisboa, Portugal.

13. Pontes $\mathrm{S}$ (2003). Caracterizar o Estado de Aptidão Física e Composição Corporal, em Dois Momentos Diferenciados, em Raparigas dos 10 aos 18 anos. Tese não publicada, Universidade Técnica de Lisboa - Faculdade de Motricidade Humana, Lisboa, Portugal. 